\title{
Chapter 3 \\ Comparing Proteomics of NCoV 19 and MERS Corona Virus
}

In order to develop any diagnostic or therapeutic protocol for COVID19 it is necessary to study the genome and proteome of the $\mathrm{NCoV}$ and annotate the proteins to identify its phylogeny and evolutionary relation with the other known organisms. The SARS $\mathrm{NCoV}$ and MERS Corona Virus belong to the Corona group of viruses and both are known to cause Respiratory illness. There is a very close similarity in the disease manifestation and mode of transmission in MERS and COVID forming basis for the comparison between the two organisms. Hence the proteins of novel Corona virus are compared with those of MERS Corona Virus (Fig. 3.1).

Inference: The above BLAST result shows that only $14 \%(\mathbf{1 3 , 6 7 0 - 1 7 , 8 6 1 )}$ of the Query genome can be aligned with the MERS Corona Virus genome (Middle East respiratory syndrome-related coronavirus isolate Bat-CoV/P.khulii/Italy/20664563/2011, complete genome: MG596803.1 [1]). However the identity in the aligned region is $70.58 \%$. This reveals a close evolutionary relation between the two organisms.

The aligned gene region with $14 \%$ of the query gene region was from 13,670 to 17,861(4192 bp) in MERS CoV which is found to share the similarity with Novel Corona Virus 2019. This region codes for ORF1ab poly protein. Thus it can be concluded that the only region that shares the highest identity between MERS CoV and Novel Corona virus 2019 is the ORF1ab polyprotein. 


\begin{tabular}{|c|c|c|c|c|c|c|c|c|}
\hline \multicolumn{2}{|r|}{ Sequences producing significant alignments } & Download \ulcorner & \multicolumn{3}{|c|}{ Manage Columns } & $\checkmark$ & how & $00 \vee$ \\
\hline \multicolumn{2}{|r|}{ select all 12 sequences selected } & & \multicolumn{2}{|c|}{ Genbank } & \multicolumn{2}{|c|}{ Graphics } & \multicolumn{2}{|c|}{ Distance tree of results } \\
\hline & Description & & $\begin{array}{l}\text { Maxx } \\
\text { Score }\end{array}$ & $\begin{array}{l}\text { Total } \\
\text { Sccore }\end{array}$ & $\begin{array}{l}\text { Overy } \\
\text { Cover }\end{array}$ & $\underset{\text { value }}{E}$ & $\begin{array}{l}\text { Per. } \\
\text { loent }\end{array}$ & Accession \\
\hline $\boldsymbol{\nabla}$ & 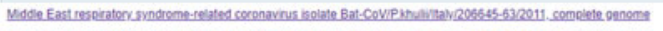 & & 767 & 767 & $14 \%$ & 0.0 & $70.56 \%$ & Mo5968031 \\
\hline 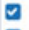 & 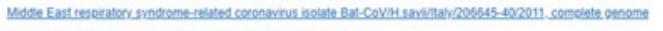 & & 765 & 765 & $13 \%$ & 0.0 & $7068 \%$ & Mos96en21 \\
\hline ص & 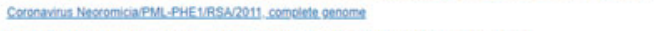 & & 758 & 945 & $14 \%$ & 00 & $70.93 \%$ & Kcasest8 4 \\
\hline $\boldsymbol{\theta}$ & 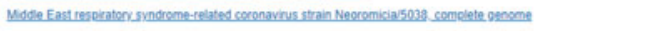 & & 730 & 912 & 1446 & 0.0 & $70.83 \%$ & MF593268 1 \\
\hline 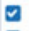 & 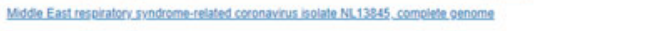 & & 699 & 689 & $13 \%$ & 0.0 & $7024 \%$ & Mo0214511. \\
\hline $\boldsymbol{\nabla}$ & 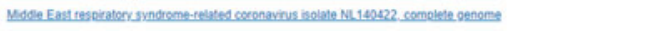 & & 632 & 632 & 115 & $26-179$ & $70.46 \%$ & v00214521 \\
\hline 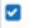 & 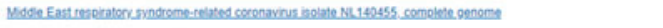 & & 628 & 628 & $12 \%$ & $20 \cdot 178$ & $70.30 \%$ & Meg97421.1 \\
\hline $\boldsymbol{\nabla}$ & 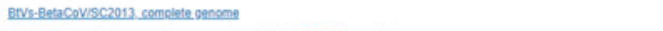 & & 542 & 542 & $11 \%$ & 3e-152 & $70.15 \%$ & K.14739211 \\
\hline $\boldsymbol{\nabla}$ & 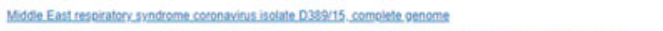 & & 442 & 442 & $5 \%$ & 30.122 & $7203 \%$ & $10 \times 1039421$ \\
\hline $\boldsymbol{\nabla}$ & 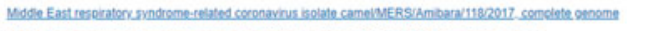 & & 178 & 178 & 15 & $10 \cdot 42$ & $7265 \%$ & 19xs4474.1 \\
\hline $\boldsymbol{\nabla}$ & 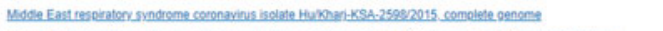 & & 178 & 178 & 15 & $10-42$ & $72.65 \%$ & kтe0s0531 \\
\hline ص & 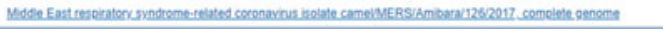 & & 172 & 172 & 15 & Se-41 & $72.48 \%$ & MxS44475.1 \\
\hline
\end{tabular}

Fig. 3.1 BLAST analysis of the whole genome sequence of novel corona virus with the MERS $\mathrm{CoV}$ genome

\subsection{ORF1ab Protein Sequence comparison of $\mathrm{NCoV}$ and MERS CoV}

MESLVPGFNEKTHVQLSLPVLQVRDVLVRGFGDSVEEVLSEARQ

HLKDGTCGLVEVEKGVLPQLEQPYVFIKRSDARTAPHGHVMVELVAELEGIQYGRSGE TLGVLVPHVGEIPVAYRKVLLRKNGNKGAGGHSYGADLKSFDLGDELGTDPYEDFQEN WNTKHSSGVTRELMRELNGGAYTRYVDNNFCGPDGYPLECIKDLLARAGKASCTLSEQ LDFIDTKRGVYCCREHEHEIAWYTERSEKSYELQTPFEIKLAKKFDTFNGECPNFVFP LNSIIKTIQPRVEKKKLDGFMGRIRSVYPVASPNECNQMCLSTLMKCDHCGETSWQTG DFVKATCEFCGTENLTKEGATTCGYLPQNAVVKIYCPACHNSEVGPEHSLAEYHNESG LKTILRKGGRTIAFGGCVFSYVGCHNKCAYWVPRASANIGCNHTGVVGEGSEGLNDNL LEILQKEKVNINIVGDFKLNEEIAIILASFSASTSAFVETVKGLDYKAFKQIVESCGN FKVTKGKAKKGAWNIGEQKSILSPLYAFASEAARVVRSIFSRTLETAQNSVRVLQKAA ITILDGISQYSLRLIDAMMFTSDLATNNLVVMAYITGGVVQLTSQWLTNIFGTVYEKL KPVLDWLEEKFKEGVEFLRDGWEIVKFISTCACEIVGGQIVTCAKEIKESVQTFFKLV NKFLALCADSIIIGGAKLKALNLGETFVTHSKGLYRKCVKSREETGLLMPLKAPKEI FLEGETLPTEVLTEEVVLKTGDLQPLEQPTSEAVEAPLVGTPVCINGLMLLEIKDTEK YCALAPNMMVTNNTFTLKGGAPTKVTFGDDTVIEVQGYKSVNITFELDERIDKVLNEK CSAYTVELGTEVNEFACVVADAVIKTLQPVSELLTPLGIDLDEWSMATYYLFDESGEF KLASHMYCSFYPPDEDEEEGDCEEEEFEPSTQYEYGTEDDYQGKPLEFGATSAALQPE EEQEEDWLDDDSQQTVGQQDGSEDNQTTTIQTIVEVQPQLEMELTPVVQTIEVNSFSG YLKLTDNVYIKNADIVEEAKKVKPTVVVNAANVYLKHGGGVAGALNKATNNAMQVESD 
DYIATNGPLKVGGSCVLSGHNLAKHCLHVVGPNVNKGEDIQLLKSAYENFNQHEVLLA PLLSAGIFGADPIHSLRVCVDTVRTNVYLAVFDKNLYDKLVSSFLEMKSEKQVEQKIA EIPKEEVKPFITESKPSVEQRKQDDKKIKACVEEVTTTLEETKFLTENLLLYIDINGN LHPDSATLVSDIDITFLKKDAPYIVGDVVQEGVLTAVVIPTKKAGGTTEMLAKALRKV PTDNYITTYPGQGLNGYTVEEAKTVLKKCKSAFYILPSIISNEKQEILGTVSWNLREM LAHAEETRKLMPVCVETKAIVSTIQRKYKGIKIQEGVVDYGARFYFYTSKTTVASLIN TLNDLNETLVTMPLGYVTHGLNLEEAARYMRSLKVPATVSVSSPDAVTAYNGYL KTPEEHFIETISLAGSYKDWSYSGQSTQLGIEFLKRGDKSVYYTSNPTTFHLDGEVIT FDNLKTLLSLREVRTIKVFTTVDNINLHTQVVDMSMTYGQQFGPTYLDGADVTKIKPH NSHEGKTFYVLPNDDTLRVEAFEYYHTTDPSFLGRYMSALNHTKKWKYPQVNGLTSIK WADNNCYLATALLTLQQIELKFNPPALQDAYYRARAGEAANFCALILAYCNKTVGELG DVRETMSYLFQHANLDSCKRVLNVVCKTCGQQQTTLKGVEAVMYMGTLSYEQFKKGVQ IPCTCGKQATKYLVQQESPFVMMSAPPAQYELKHGTFTCASEYTGNYQCGHYKHITSK ETLYCIDGALLTKSSEYKGPITDVFYKENSYTTTIKPVTYKLDGVVCTEIDPKLDNYY KKDNSYFTEQPIDLVPNQPYPNASFDNFKFVCDNIKFADDLNQLTGYKKPASRELKVT FFPDLNGDVVAIDYKHYTPSFKKGAKLLHKPIVWHVNNATNKATYKPNTWCIRCLWST KPVETSNSFDVLKSEDAQGMDNLACEDLKPVSEEVVENPTIQKDVLECNVKTTEVVGD IILKPANNSLKITEEVGHTDLMAAYVDNSSLTIKKPNELSRVLGLKTLATHGLAAVNS VPWDTIANYAKPFLNKVVSTTTNIVTRCLNRVCTNYMPYFFTLLLQLCTFTRSTNSRI

KASMPTTIAKNTVKSVGKFCLEASFNYLKSPNFSKLINIIIWFLLLSVCLGSLIYSTA ALGVLMSNLGMPSYCTGYREGYLNSTNVTIATYCTGSIPCSVCLSGLDSLDTYPSLET IQITISSFKWDLTAFGLVAEWFLAYILFTRFFYVLGLAAIMQLFFSYFAVHFISNSWL MWLIINLVQMAPISAMVRMYIFFASFYYVWKSYVHVVDGCNSSTCMMCYKRNRATRVE CTTIVNGVRRSFYVYANGGKGFCKLHNWNCVNCDTFCAGSTFISDEVARDLSLQFKRP INPTDQSSYIVDSVTVKNGSIHLYFDKAGQKTYERHSLSHFVNLDNLRANNTKGSLPI NVIVFDGKSKCEESSAKSASVYYSQLMCQPILLLDQALVSDVGDSAEVAVKMFDAYVN TFSSTFNVPMEKLKTLVATAEAELAKNVSLDNVLSTFISAARQGFVDSDVETKDVVEC LKLSHQSDIEVTGDSCNNYMLTYNKVENMTPRDLGACIDCSARHINAQVAKSHNIALI WNVKDFMSLSEQLRKQIRSAAKKNNLPFKLTCATTRQVVNVVTTKIALKGGKIVNNWL KQLIKVTLVFLFVAAIFYLITPVHVMSKHTDFSSEIIGYKAIDGGVTRDIASTDTCFA NKHADFDTWFSQRGGSYTNDKACPLIAAVITREVGFVVPGLPGTILRTTNGDFLHFLP RVFSAVGNICYTPSKLIEYTDFATSACVLAAECTIFKDASGKPVPYCYDTNVLEGSVA 
YESLRPDTRYVLMDGSIIQFPNTYLEGSVRVVTTFDSEYCRHGTCERSEAGVCVSTSG RWVLNNDYYRSLPGVFCGVDAVNLLTNMFTPLIQPIGALDISASIVAGGIVAIVVTCL AYYFMRFRRAFGEYSHVVAFNTLLFLMSFTVLCLTPVYSFLPGVYSVIYLYLTFYLTN DVSFLAHIQWMVMFTPLVPFWITIAYIICISTKHFYWFFSNYLKRRVVFNGVSFSTFE EAALCTFLLNKEMYLKLRSDVLLPLTQYNRYLALYNKYKYFSGAMDTTSYREAACCHL AKALNDFSNSGSDVLYQPPQTSITSAVLQSGFRKMAFPSGKVEGCMVQVTCGTTTLNG LWLDDVVYCPRHVICTSEDMLNPNYEDLLIRKSNHNFLVQAGNVQLRVIGHSMQNCVL KLKVDTANPKTPKYKFVRIQPGQTFSVLACYNGSPSGVYQCAMRPNFTIKGSFLNGSC GSVGFNIDYDCVSFCYMHHMELPTGVHAGTDLEGNFYGPFVDRQTAQAAGTDTTITVN VLAWLYAAVINGDRWFLNRFTTTLNDFNLVAMKYNYEPLTQDHVDILGPLSAQTGIAV LDMCASLKELLQNGMNGRTILGSALLEDEFTPFDVVRQCSGVTFQSAVKRTIKGTHHW LLLTILTSLLVLVQSTQWSLFFFLYENAFLPFAMGIIAMSAFAMMFVKHKHAFLCLFL LPSLATVAYFNMVYMPASWVMRIMTWLDMVDTSLSGFKLKDCVMYASAVVLLILMTAR TVYDDGARRVWTLMNVLTLVYKVYYGNALDQAISMWALIISVTSNYSGVVTTVMFLAR GIVFMCVEYCPIFFITGNTLQCIMLVYCFLGYFCTCYFGLFCLLNRYFRLTLGVYDYL VSTQEFRYMNSQGLLPPKNSIDAFKLNIKLLGVGGKPCIKVATVQSKMSDVKCTSVVL LSVLQQLRVESSSKLWAQCVQLHNDILLAKDTTEAFEKMVSLLSVLLSMQGAVDINKL CEEMLDNRATLQAIASEFSSLPSYAAFATAQEAYEQAVANGDSEVVLKKLKKSLNVAK SEFDRDAAMQRKLEKMADQAMTQMYKQARSEDKRAKVTSAMQTMLFTMLRKLDNDALN NIINNARDGCVPLNIIPLTTAAKLMVVIPDYNTYKNTCDGTTFTYASALWEIQQVVDA DSKIVQLSEISMDNSPNLAWPLIVTALRANSAVKLQNNELSPVALRQMSCAAGTTQTA CTDDNALAYYNTTKGGRFVLALLSDLQDLKWARFPKSDGTGTIYTELEPPCRFVTDTP KGPKVKYLYFIKGLNNLNRGMVLGSLAATVRLQAGNATEVPANSTVLSFCAFAVDAAK AYKDYLASGGQPITNCVKMLCTHTGTGQAITVTPEANMDQESFGGASCCLYCRCHIDH PNPKGFCDLKGKYVQIPTTCANDPVGFTLKNTVCTVCGMWKGYGCSCDQLREPMLQSA DAQSFLNRVCGVSAARLTPCGTGTSTDVVYRAFDIYNDKVAGFAKFLKTNCCRFQEKD EDDNLIDSYFVVKRHTFSNYQHEETIYNLLKDCPAVAKHDFFKFRIDGDMVPHISRQR LTKYTMADLVYALRHFDEGNCDTLKEILVTYNCCDDDYFNKKDWYDFVENPDILRVYA NLGERVRQALLKTVQFCDAMRNAGIVGVLTLDNQDLNGNWYDFGDFIQTTPGSGVPVV DSYYSLLMPILTLTRALTAESHVDTDLTKPYIKWDLLKYDFTEERLKLFDRYFKYWDQ TYHPNCVNCLDDRCILHCANFNVLFSTVFPPTSFGPLVRKIFVDGVPFVVSTGYHFRE LGVVHNQDVNLHSSRLSFKELLVYAADPAMHAASGNLLLDKRTTCFSVAALTNNVAFQ TVKPGNFNKDFYDFAVSKGFFKEGSSVELKHFFFAQDGNAAISDYDYYRYNLPTMCDI RQLLFVVEVVDKYFDCYDGGCINANQVIVNNLDKSAGFPFNKWGKARLYYDSMSYEDQ DALFAYTKRNVIPTITQMNLKYAISAKNRARTVAGVSICSTMTNRQFHQKLLKSIAAT RGATVVIGTSKFYGGWHNMLKTVYSDVENPHLMGWDYPKCDRAMPNMLRIMASLVLAR 
KHTTCCSLSHRFYRLANECAQVLSEMVMCGGSLYVKPGGTSSGDATTAYANSVFNICQ AVTANVNALLSTDGNKIADKYVRNLQHRLYECLYRNRDVDTDFVNEFYAYLRKHFSMM ILSDDAVVCFNSTYASQGLVASIKNFKSVLYYQNNVFMSEAKCWTETDLTKGPHEFCS QHTMLVKQGDDYVYLPYPDPSRILGAGCFVDDIVKTDGTLMIERFVSLAIDAYPLTKH PNQEYADVFHLYLQYIRKLHDELTGHMLDMYSVMLTNDNTSRYWEPEFYEAMYTPHTV LQAVGACVLCNSQTSLRCGACIRRPFLCCKCCYDHVISTSHKLVLSVNPYVCNAPGCD VTDVTQLYLGGMSYYCKSHKPPISFPLCANGQVFGLYKNTCVGSDNVTDFNAIATCDW TNAGDYILANTCTERLKLFAAETLKATEETFKLSYGIATVREVLSDRELHLSWEVGKP RPPLNRNYVFTGYRVTKNSKVQIGEYTFEKGDYGDAVVYRGTTTYKLNVGDYFVLTSH TVMPLSAPTLVPQEHYVRITGLYPTLNISDEFSSNVANYQKVGMQKYSTLQGPPGTGK SHFAIGLALYYPSARIVYTACSHAAVDALCEKALKYLPIDKCSRIIPARARVECFDKF KVNSTLEQYVFCTVNALPETTADIVVFDEISMATNYDLSVVNARLRAKHYVYIGDPAQ LPAPRTLLTKGTLEPEYFNSVCRLMKTIGPDMFLGTCRRCPAEIVDTVSALVYDNKLK AHKDKSAQCFKMFYKGVITHDVSSAINRPQIGVVREFLTRNPAWRKAVFISPYNSQNA VASKILGLPTQTVDSSQGSEYDYVIFTQTTETAHSCNVNRFNVAITRAKVGILCIMSD RDLYDKLQFTSLEIPRRNVATLQAENVTGLFKDCSKVITGLHPTQAPTHLSVDTKFKT EGLCVDIPGIPKDMTYRRLISMMGFKMNYQVNGYPNMFITREEAIRHVRAWIGFDVEG CHATREAVGTNLPLQLGFSTGVNLVAVPTGYVDTPNNTDFSRVSAKPPPGDQFKHLIP LMYKGLPWNVVRIKIVQMLSDTLKNLSDRVVFVLWAHGFELTSMKYFVKIGPERTCCL CDRRATCFSTASDTYACWHHSIGFDYVYNPFMIDVQQWGFTGNLQSNHDLYCQVHGNA HVASCDAIMTRCLAVHECFVKRVDWTIEYPIIGDELKINAACRKVQHMVVKAALLADK FPVLHDIGNPKAIKCVPQADVEWKFYDAQPCSDKAYKIEELFYSYATHSDKFTDGVCL FWNCNVDRYPANSIVCRFDTRVLSNLNLPGCDGGSLYVNKHAFHTPAFDKSAFVNLKQ LPFFYYSDSPCESHGKQVVSDIDYVPLKSATCITRCNLGGAVCRHHANEYRLYLDAYN MMISAGFSLWVYKQFDTYNLWNTFTRLQSLENVAFNVVNKGHFDGQQGEVPVSIINNT VYTKVDGVDVELFENKTTLPVNVAFELWAKRNIKPVPEVKILNNLGVDIAANTVIWDY KRDAPAHISTIGVCSMTDIAKKPTETICAPLTVFFDGRVDGQVDLFRNARNGVLITEG SVKGLQPSVGPKQASLNGVTLIGEAVKTQFNYYKKVDGVVQQLPETYFTQSRNLQEFK PRSQMEIDFLELAMDEFIERYKLEGYAFEHIVYGDFSHSQLGGLHLLIGLAKRFKESP FELEDFIPMDSTVKNYFITDAQTGSSKCVCSVIDLLLDDFVEIIKSQDLSVVSKVVKV TIDYTEISFMLWCKDGHVETFYPKLQSSQAWQPGVAMPNLYKMQRMLLEKCDLQNYGD SATLPKGIMMNVAKYTQLCQYLNTLTLAVPYNMRVIHFGAGSDKGVAPGTAVLRQWLP TGTLLVDSDLNDFVSDADSTLIGDCATVHTANKWDLIISDMYDPKTKNVTKENDSKEG FFTYICGFIQQKLALGGSVAIKITEHSWNADLYKLMGHFAWWTAFVTNVNASSSEAFL IGCNYLGKPREQIDGYVMHANYIFWRNTNPIQLSSYSLFDMSKFPLKLRGTAVMSLKE GQINDMILSLLSKGRLIIRENNRVVISSDVLVNN

The above is the Proteomic sequence of ORF1AB of NCoV where, Yellow residues-Corona Rpol $\mathrm{N}$ domain

Green residues-Viral Helicase

Yellow + cyan + Green residues-Region sharing identity to the MERS CoV Proteome (Fig. 3.2). 


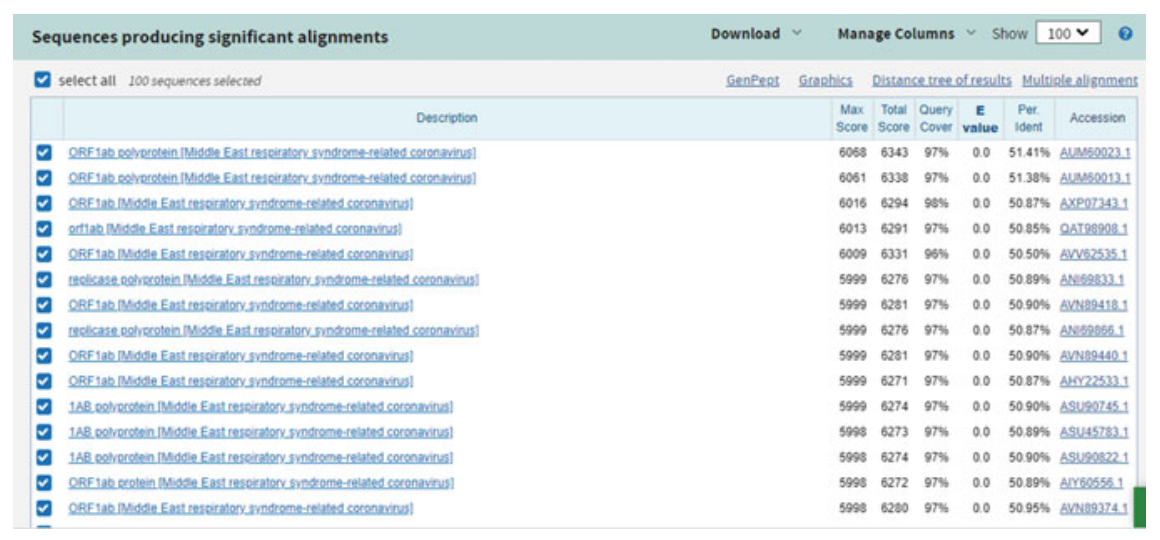

Fig. 3.2 Comparison of the ORF1ab polyprotein with the MERS CoV proteome using BLAST

Inference: The above result shows that ORF1AB protein of query (Novel Corona Virus 2019) shares $51.41 \%$ identity to that of the MERS CoV. Thus they both share a close evolutionary relation.

\subsection{Comparison of Spike (S) Proteins of NCoV and MERS CoV}

\section{S protein of Novel CoV2019:}

MFVFLVLLPLVSSQCVNLTTRTQLPPAYTNSFTRGVYYPDKVFR SSVLHSTQDLFLPFFSNVTWFHAIHVSGTNGTKRFDNPVLPFNDGVYFASTEKSNIIR GWIFGTTLDSKTQSLLIVNNATNVVIKVCEFQFCNDPFLGVYYHKNNKSWMESEFRVY SSANNCTFEYVSQPFLMDLEGKQGNFKNLREFVFKNIDGYFKIYSKHTPINLVRDLPQ GFSALEPLVDLPIGINITRFQTLLALHRSYLTPGDSSSGWTAGAAAYYVGYLQPRTFL LKYNENGTITDAVDCALDPLSETKCTLKSFTVEKGIYQTSNFRVQPTESIVRFPNITN LCPFGEVFNATRFASVYAWNRKRISNCVADYSVLYNSASFSTFKCYGVSPTKLNDLCF TNVYADSFVIRGDEVRQIAPGQTGKIADYNYKLPDDFTGCVIAWNSNNLDSKVGGNYN YLYRLFRKSNLKPFERDISTEIYQAGSTPCNGVEGFNCYFPLQSYGFQPTNGVGYQPY RVVVLSFELLHAPATVCGPKKSTNLVKNKCVNFNFNGLTGTGVLTESNKKFLPFQQFG RDIADTTDAVRDPQTLEILDITPCSFGGVSVITPGTNTSNQVAVLYQDVNCTEVPVAI HADQLTPTWRVYSTGSNVFQTRAGCLIGAEHVNNSYECDIPIGAGICASYQTQTNSPR RARSVASQSIIAYTMSLGAENSVAYSNNSIAIPTNFTISVTTEILPVSMTKTSVDCTM YICGDSTECSNLLLQYGSFCTQLNRALTGIAVEQDKNTQEVFAQVKQIYKTPPIKDFG GFNFSQILPDPSKPSKRSFIEDLLFNKVTLADAGFIKQYGDCLGDIAARDLICAQKFN GLTVLPPLLTDEMIAQYTSALLAGTITSGWTFGAGAALQIPFAMQMAYRFNGIGVTQN VLYENQKLIANQFNSAIGKIQDSLSSTASALGKLQDVVNQNAQALNTLVKQLSSNFGA ISSVLNDILSRLDKVEAEVQIDRLITGRLQSLQTYVTQQLIRAAEIRASANLAATKMS ECVLGQSKRVDFCGKGYHLMSFPQSAPHGVVFLHVTYVPAQEKNFTTAPAICHDGKAH FPREGVFVSNGTHWFVTQRNFYEPQIITTDNTFVSGNCDVVIGIVNNTVYDPLQPELD SFKEELDKYFKNHTSPDVDLGDISGINASVVNIQKEIDRLNEVAKNLNESLIDLQELG KYEQYIKWPWYIWLGFIAGLIAIVMVTIMLCCMTSCCSCLKGCCSCGSCCKFDEDDSEPVLKGVKLHYT 


\begin{tabular}{|c|c|c|c|c|c|c|c|c|}
\hline \multicolumn{2}{|r|}{ Sequences producing significant alignments } & Download & \multicolumn{4}{|c|}{ Manage Columns $\checkmark$} & Show & $100 \vee$ \\
\hline \multicolumn{2}{|r|}{ D select all 100 sequences selected } & Genpees & \multicolumn{6}{|c|}{ Graphics Distance tree of results Multiple alisoment } \\
\hline & Description & & $\begin{array}{l}\text { Max } \\
\text { Scoxe }\end{array}$ & $\begin{array}{l}\text { Total } \\
\text { Score }\end{array}$ & $\begin{array}{l}\text { Ouery } \\
\text { Cover }\end{array}$ & $\underset{\text { value }}{E}$ & $\begin{array}{l}\text { Per. } \\
\text { ident }\end{array}$ & Accession \\
\hline ص & 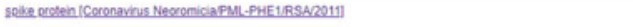 & & 571 & 571 & $83 \%$ & 0.0 & $34.55 \%$ & AOCY296502 \\
\hline a & 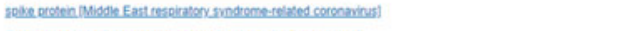 & & 570 & 570 & $83 \%$ & 0.0 & $34.46 \%$ & ATO393921 \\
\hline 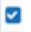 & 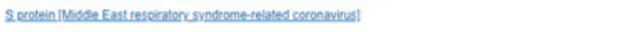 & & 560 & 560 & $79 \%$ & $10-177$ & $35.57 \%$ & aWHesses4 1 \\
\hline ص & 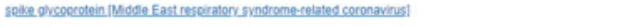 & & 553 & 553 & $78 \%$ & $20-177$ & $35.10 \%$ & ASH266101 \\
\hline $\boldsymbol{\nabla}$ & 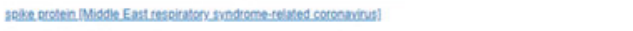 & & 555 & 555 & $78 \%$ & 8e-176 & $3529 \%$ & QAT28sas \\
\hline ص & 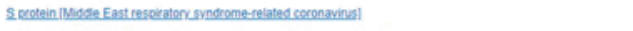 & & 555 & 555 & $78 \%$ & 16-175 & $35.19 \%$ & AnN893131 \\
\hline ص & 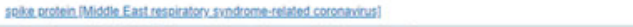 & & 555 & sss & $78 \%$ & $20-175$ & $35.19 \%$ & ALA49374.1 \\
\hline ص & 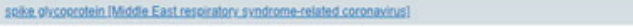 & & 555 & 555 & $78 \%$ & $2 e-175$ & $35.19 \%$ & ASU90241.1 \\
\hline ص & 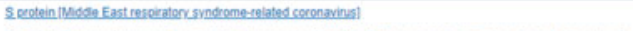 & & 554 & 584 & $78 \%$ & $20-175$ & $35.19 \%$ & AnN892911 \\
\hline ص & 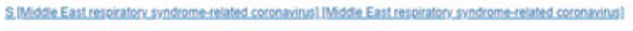 & & 554 & 554 & $78 \%$ & 20-175 & $3529 \%$ & A:C441021 \\
\hline ص & 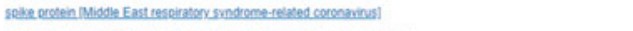 & & 554 & 554 & $78 \%$ & 2e-175 & $35.13 \%$ & ALL544521 \\
\hline ص & 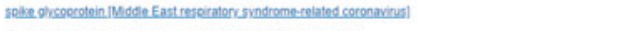 & & 554 & SS4 & $78 \%$ & 3e-175 & $35.19 \%$ & AM0034011 \\
\hline ص & 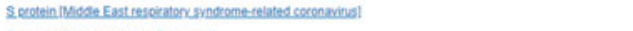 & & 554 & 554 & $78 \%$ & 3e-175 & $35.19 \%$ & Atraz25451 \\
\hline ص & Serotein IBetacconavinus Enotand 11 & & 554 & 554 & $78 \%$ & 3e-175 & $35.19 \%$ & YP 0071885791 \\
\hline 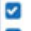 & 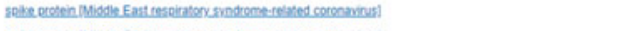 & & 554 & 554 & $78 \%$ & 3e-175 & $35.19 \%$ & BLA493951 \\
\hline ص & 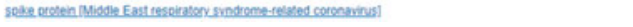 & & 554 & 554 & $78 \%$ & $3 e-175$ & $35.19 \%$ & Alos50371 \\
\hline
\end{tabular}

Fig. 3.3 Comparison of the S Protein with MERS CoV proteome using BLAST

Inference: From the above BLAST result it can be inferred that the $\mathrm{S}$ protein of Query shows an identity of about $34 \%$ to the MERS CoV Spike protein (Fig. 3.3).

\title{
3.3 Comparison of Membrane (M) Proteins of NCoV and MERS CoV
}

\author{
MADSNGTITVEELKKLLEQWNLVIGFLFLTWICLLQFAYANRNR \\ FLYIIKLIFLWLLWPVTLACFVLAAVYRINWITGGIAIAMACLVGLMWLSYFIASFRL \\ FARTRSMWSFNPETNILLNVPLHGTILTRPLLESELVIGAVILRGHLRIAGHHLGRCD \\ IKDLPKEITVATSRTLSYYKLGASQRVAGDSGFAAYSRYRIGNYKLNTDHSSSSDNIALLVQ
}

\begin{tabular}{|c|c|c|c|c|c|c|c|c|}
\hline \multicolumn{2}{|r|}{ Sequences producing significant atignments } & \multirow{2}{*}{$\begin{array}{l}\text { Downlosd } ~ \\
\text { Genfrest }\end{array}$} & \multicolumn{4}{|c|}{ Manage Columns $\checkmark$} & \multirow{2}{*}{ Show } & \multirow{2}{*}{$100 \mathrm{~V} 0$} \\
\hline \multicolumn{2}{|c|}{ D select all osequences seilected } & & Grastics & $\cos$ & rance ti & veredire & & \\
\hline & Dencripton & & $\begin{array}{l}\text { Max } \\
\text { Scose }\end{array}$ & $\begin{array}{l}\text { Toed } \\
\text { Secoce }\end{array}$ & $\begin{array}{l}\text { Ouvy } \\
\text { Cover }\end{array}$ & $\underset{\text { value }}{\text { E }}$ & $\begin{array}{l}\text { Pew } \\
\text { Went }\end{array}$ & Accession \\
\hline ○ & 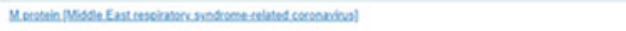 & & 189 & 169 & $90 \mathrm{~s}$ & $40-61$ & $4505 \%$ & anassene 1 \\
\hline$\square$ & 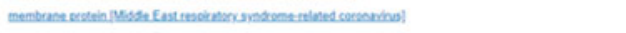 & & 168 & 160 & sos & $\operatorname{los} 1$ & 4065 & suseseres \\
\hline 0 & 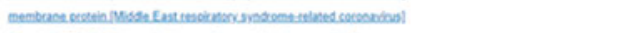 & & 160 & 168 & sos & 1060 & 44065 & sumeseazes \\
\hline Q & 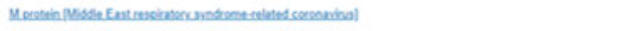 & & 107 & 107 & sos & 3060 & 4075 & $\sin 52532.1$ \\
\hline$\square$ & 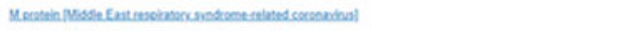 & & 166 & tes & sos & 4060 & usss & anassere1 \\
\hline$\square$ & 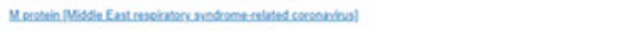 & & 106 & tes & sos & 10.59 & 4soss & Ans:25424 \\
\hline ○ & 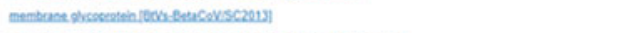 & & 105 & ves & sos & $20-59$ & sors & 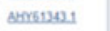 \\
\hline Q & 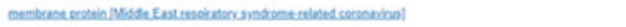 & & 161 & 161 & sos & $10-57$ & 42065 & atrosuts: \\
\hline$\square$ & 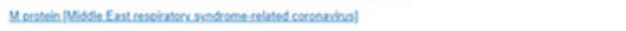 & & 100 & 100 & sos & 1657 & 4355 & acoversen \\
\hline$\square$ & 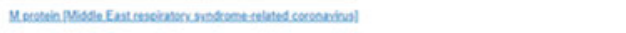 & & 100 & 160 & sos & 2057 & 43ss & ansesse.1 \\
\hline 0 & 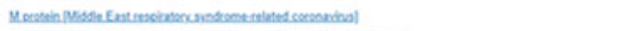 & & $m$ & 179 & 905 & $20-59$ & 42065 & anceren:1 \\
\hline ○ & 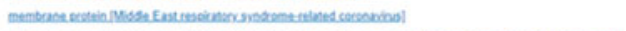 & & 179 & 179 & sos & $30-59$ & $220 s \mathrm{~s}$ & Asuev214: \\
\hline 0 & 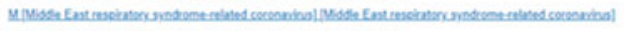 & & 19 & 178 & sos & 3057 & 42065 & ARZ4465: \\
\hline$\underset{m}{\mathrm{O}}$ & 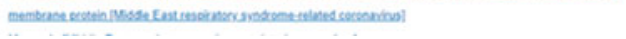 & & 179 & 179 & sos & $30-57$ & $22 \times 5$ & ALTEGose.t \\
\hline
\end{tabular}

Fig. 3.4 Comparison of the M Protein with MERS CoV proteome using BLAST 
Inference: The above result shows that the query $M$ protein shares $45.05 \%$ identity to MERS CoV M protein. This can impart some minor variation in the Membrane protein structure between the two pathogens (Fig. 3.4).

\subsection{Comparison of Envelope (E) Proteins of $\mathrm{NCoV}$ and MERS CoV}

\begin{tabular}{|c|c|c|c|c|c|c|c|}
\hline & Description & $\begin{array}{l}\text { Masx } \\
\text { Scorere }\end{array}$ & $\begin{array}{l}\text { Total } \\
\text { Score }\end{array}$ & $\begin{array}{l}\text { Ouery } \\
\text { cover }\end{array}$ & $\begin{array}{c}\mathbf{E} \\
\text { value }\end{array}$ & $\begin{array}{l}\text { Per. } \\
\text { Ideent }\end{array}$ & Accession \\
\hline ص & 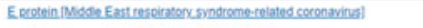 & 51.2 & $\$ 1.2$ & $94 \%$ & $80-11$ & $40.00 \%$ & Awat6594.1 \\
\hline ( & 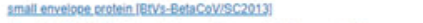 & 50.8 & 50.8 & 94\% & 10-10 & $38.67 \%$ & AHY813421 \\
\hline $\boldsymbol{\theta}$ & 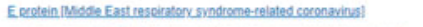 & 49.7 & 49.7 & $94 \%$ & 4e-10 & $37.33 \%$ & AVN625621 \\
\hline ఐ & 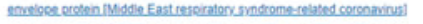 & 49.3 & 49.3 & $94 \%$ & $40 \cdot 10$ & $37.33 \%$ & AUM60019.1 \\
\hline 正 & 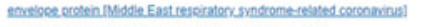 & 48.5 & 48.5 & $94 \%$ & 9e-10 & $37.33 \%$ & ATO29391.1 \\
\hline 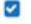 & 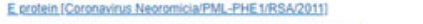 & 47.4 & 47.4 & $94 \%$ & $3 e-09$ & $36.00 \%$ & Al013101.1. \\
\hline 正 & 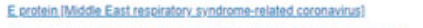 & 46.6 & 456 & $94 \%$ & $5 e-09$ & $36.00 \%$ & AVNE25311. \\
\hline ص & 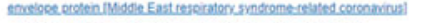 & 45.8 & 45.8 & $94 \%$ & 10.08 & $36.00 \%$ & ALA493921 \\
\hline 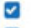 & 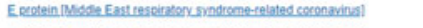 & 45.1 & 45.1 & $94 \%$ & $2 \mathrm{e}-0 \mathrm{~s}$ & $3600 \%$ & Aovoles721 \\
\hline $\boldsymbol{0}$ & 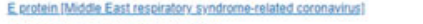 & 45.1 & 45.1 & $94 \%$ & $20-08$ & $36.00 \%$ & AHz656231 \\
\hline 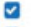 & Ereotein/Betacoronainus Enoland 11 & 45.1 & 45.1 & $94 \%$ & $2 \mathrm{e}-08$ & $3600 \%$ & YP 0071805841 \\
\hline ఐ & 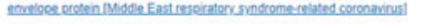 & 45.1 & 45.1 & $94 \%$ & 20.08 & $36.00 \%$ & Asugo334.1 \\
\hline 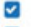 & 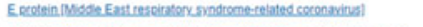 & 45.1 & 45.1 & $94 \%$ & 20.08 & $36.00 \%$ & ANF292661 \\
\hline ص & 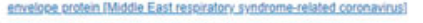 & 45.1 & 45.1 & $945 \%$ & $20-08$ & $36.00 \%$ & ALR69545:1 \\
\hline 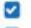 & 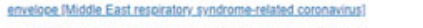 & 45.1 & 45.1 & $94 \%$ & $20-08$ & $36.00 \%$ & gem11741.1 \\
\hline 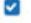 & 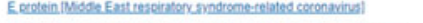 & 45.1 & 45.1 & $94 \%$ & $20-08$ & $36.00 \%$ & ALX27237.1 \\
\hline $\boldsymbol{\theta}$ & 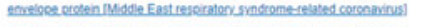 & 44.7 & 44.7 & $94 \%$ & $3 e-08$ & $36.00 \%$ & AN169:24.1 \\
\hline ఐ & 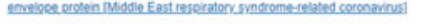 & 44.7 & 447 & 94\% & $39-08$ & $36.00 \%$ & ALA493451 \\
\hline 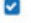 & 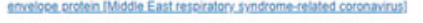 & 44.3 & 443 & 94\% & $40-08$ & $3600 \%$ & Asugess41 \\
\hline 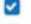 & 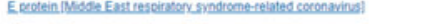 & 43.1 & 43.1 & 94\% & 10-07 & $34.67 \%$ & ATOQ45241 \\
\hline q & 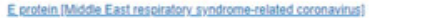 & 42.7 & 42.7 & $94 \%$ & $20-07$ & $34.67 \%$ & AOV Vos 5401 \\
\hline
\end{tabular}

Fig. 3.5 Comparison of the E Protein with MERS CoV proteome using BLAST

Inference: From the above BLAST result it can be inferred that the E protein shares a considerable similarity with the E protein of MERS CoV with query coverage of $94 \%$ and identity being $40 \%$ (Fig. 3.5). 


\title{
3.5 Comparison of Nucleocapsid (N) Proteins of NCoV and MERS CoV
}

\author{
MSDNGPQNQRNAPRITFGGPSDSTGSNQNGERSGARSKQRRPQG \\ LPNNTASWFTALTQHGKEDLKFPRGQGVPINTNSSPDDQIGYYRRATRRIRGGDGKMK \\ DLSPRWYFYYLGTGPEAGLPYGANKDGIIWVATEGALNTPKDHIGTRNPANNAAIVLQ \\ LPQGTTLPKGFYAEGSRGGSQASSRSSSRSRNSSRNSTPGSSRGTSPARMAGNGGDAA \\ LALLLLDRLNQLESKMSGKGQQQQGQTVTKKSAAEASKKPRQKRTATKAYNVTQAFGR \\ RGPEQTQGNFGDQELIRQGTDYKHWPQIAQFAPSASAFFGMSRIGMEVTPSGTWLTYT \\ GAIKLDDKDPNFKDQVILLNKHIDAYKTFPPTEPKKDKKKKADETQALPQRQKKQQTV \\ TLLPAADLDDFSKQLQQSMSSADSTQA
}

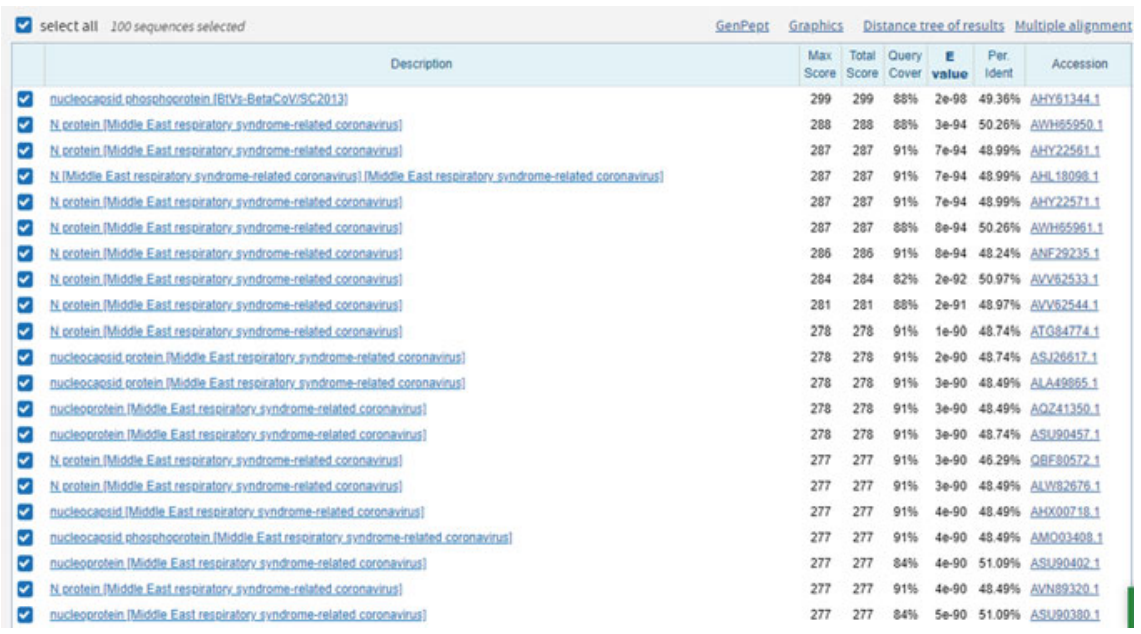

Fig. 3.6 Comparison of the N Protein with MERS CoV proteome using BLAST

Inference: The above result shows that MERS CoV Nucleocapsid shares 50.26\% identity to the $\mathrm{N}$ protein query with coverage of $88 \%$ (Fig. 3.6). 


\subsection{Conclusion}

From the above comparative analysis between the structural proteins and ORF1ab (being the longest protein in the virus) of $\mathrm{NCoV}$ and MERS Corona Virus, it can be concluded that both the organisms share a considerable similarity though not very high. However the ORF1ab is the longest protein in the Query proteome and is found to share a great similarity to that of MERS Corona Virus. Thus the study can be focused on the annotation of ORF1ab protein and targeting it for the Drug Docking studies. Further chapters of the book focus on the complete structural and functional characterization of the ORF1ab polyprotein of Novel Corona virus 2019.

\section{References}

1. Moreno A et al (2017) Detection and full genome characterization of two beta CoV viruses related to Middle East respiratory syndrome from bats in Italy. Virol J 14(1):239. https://doi. org/10.1186/s12985-017-0907-1 PMID:29258555

2. Baranov PV et al (2005) Programmed ribosomal frameshifting in decoding the SARS-CoV genome. Virology 332(2):498-510 PMID:15680415 